\title{
An Origami-Inspired Flexible Pneumatic Actuator
}

\author{
François Schmitt ${ }^{1}$, Olivier Piccin ${ }^{2}$, Laurent Barbé $^{1}$ and Bernard Bayle ${ }^{1}$
}

\begin{abstract}
This paper presents a new actuator designed to produce forces under short stroke displacements. Two variants of the prototype have been manufactured using Multi-Material Additive Manufacturing process, based on a flexible origamiinspired architecture. The structure consists of an airtight chamber constituted by rigid plates combined with flexible hinges and surfaces in order to allow the generation of motion. We propose several insights on integration issues such as limited material resistance and maximum range of motion. Both versions of the prototype are then tested to assess their performances for single strokes and cyclic loading.
\end{abstract}

\section{INTRODUCTION}

Soft materials offer new possibilities to design robot technologies well adapted to interactions with their environment, with new solutions to access confined spaces [1], grasp variable shape objects [2] and adapt to more situations [3]. Soft robots are also known for their high resilience [4], [5] and good power to weight ratio [6]. They may offer other interesting features, such as variable stiffness [7], or zero backlash thanks to monolithic designs. However, their structural softness is not well adapted to actuate conventional rigid robotic structures.

In parallel to the development of soft robotics, new manufacturing methods have spread very fast. This is in particular the case of additive manufacturing which offers nearly unlimited possibilities to build shapes that were impossible to manufacture using conventional methods. More recently, Multi-Material Additive Manufacturing (MMAM) has enabled to combine heterogeneous materials. This is especially interesting for soft robots design, as it allows mixing soft and rigid properties in a monolithic part [8], [9]. Compared to conventional plastics industry processes, it does not require complex multi-step processes such as overmolding or lamination.

Manufacturing soft robots using MMAM is not easy however. Soft robots often employ highly anisotropic materials, such as paper or fabric sheets used to reinforce the softer structure [10], [11]. The soft materials used are generally silicon polymers, exhibiting low stiffness and very high breaking elongation [12]. When using MMAM however, the designer has a limited choice in the materials and manufacturing process. Even though commercially available MMAM materials exhibit elastic behavior comparable to other classical materials, they tend to break at much lower stress levels. This drawback becomes even more pronounced when trying to scale down the parts size, as commercially

This work was supported by French state funds managed by the ANR within the Investissements d'Avenir program (Robotex ANR-10-EQPX-44, Labex CAMI - ANR-11-LABX-0004) and by the Région Grand Est. ${ }^{1} \mathrm{~F}$. Schmitt (f.schmitt@unistra.fr), L. Barbé and B. Bayle are with ICube Laboratory, University of Strasbourg-CNRS, France. ${ }^{2} \mathrm{O}$. Piccin is with ICube Laboratory, and INSA Strasbourg, France available MMAM machines generally have a manufacturing accuracy of about a tenth of millimeter.

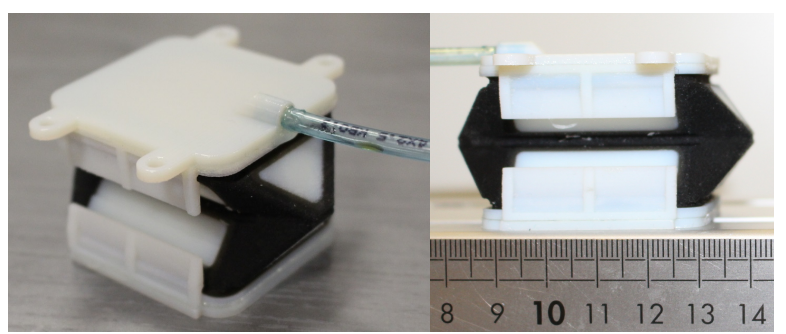

Fig. 1. General view of the actuator prototype (Version B). The ruler is graduated in centimeters.

In this paper, a new kind of soft fluidic actuator is proposed (see Fig. 1), inspired by recent developments in origami robots. It is based on a prismatic deformable pocket, built as a quasi-monolithic block using MMAM. This technology allows force generation for short stroke movements on a lightweight structure, with the motivation to develop building blocks for collaborative robotics where high power to weight ratio is more important than positioning accuracy. In this context, monolithic MMAM parts offer interesting opportunities to simplify the building process for such an actuator while keeping its footprint low.

In Section II, the geometry of the actuator as well as its parametrization are presented, followed in Section III by the study of the influence of several parameters on the theoretical performances of the actuator. The actuator implementation is then presented in Section IV, and the performances of the resulting prototypes are then assessed in Section V. Finally, in Section VI, the obtained results and the perspective of this work are discussed.

\section{DESIGN PRINCIPLES}

Origami designs have seen recent spikes in interest as they allow to build lightweight and stiff structures, while also being able to reconfigure themselves [13], [14], [15], [16]. Those properties are of particular interest in the design of fluidic actuators [17], [18], [19] as they can be compact, lightweight, offer relatively large ranges of displacement and can also exhibit large off-axis stiffness [20]. These systems have inspired the system presented in the following.

\section{A. Description and parametrization}

The geometry of the proposed actuator can be modeled as a polyhedron and is parametrized as presented in Fig. 2 . It is composed of three sub-volumes, the central one being a prismatic body obtained by the extrusion of a honeycomb pattern along the $\left(O_{0}, \mathbf{z}\right)$ axis. The other two volumes are the end caps formed by a combination of both trapezoidal and triangular faces, in a pattern similar to the origami 
"waterbomb" [21], [22]. The obtained geometry exhibits three planar symmetries thus the actuator geometry can be defined by a set of five constant parameters $(a, b, g, h, w)$ and the variable parameter $e$ describing the actuator's extension. Moreover, two variable parameters $\theta$ and $\varphi$ are introduced to describe the configuration of the prismatic shape and the two caps. When the angle $\theta>0$ (resp. $<0$ ), the prismatic part profile is convex (resp. concave) and when $\varphi>0$ (resp. $<0)$, the end caps are re-entrant (resp. salient) with respect to the prismatic volume.

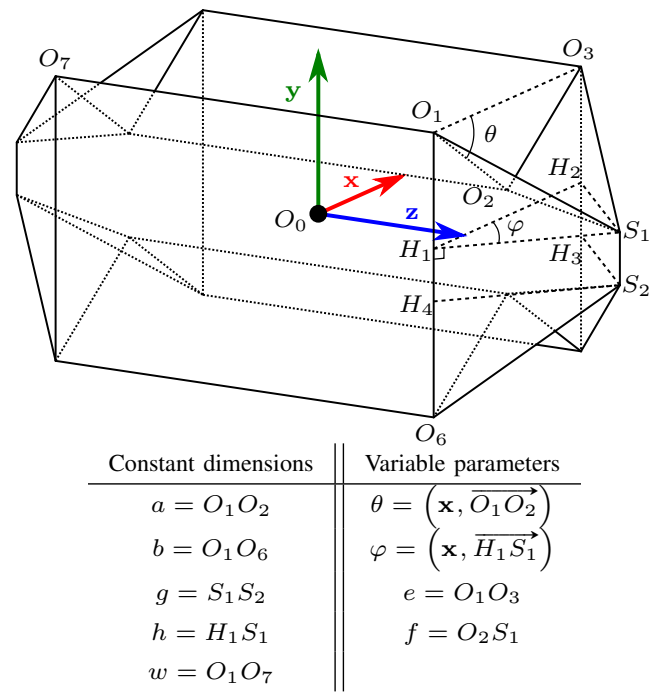

Fig. 2. Geometry and parametrization of the proposed actuator.

The design objective is to obtain an articulated polyhedron with rigid faces in which the edges act as hinges, allowing the faces to rotate with respect to their neighbors. Moreover, the polyhedron should provide a variable internal volume during its operation. However, following the bellows conjecture [23], the internal volume of an articulated polyhedron remains constant under rigid deformations and thus it is mandatory that some faces or edges of the polyhedron change their geometry during operation. In order to better guide the motion, deformations should be localized in the end caps. Thus, the length $f$ of the edge $O_{2} S_{1}$ was chosen as a variable dimension to accommodate the volume variation.

\section{B. Geometric model and deployment limits}

The deployment of the proposed polyhedron can be described using one parameter chosen among $e, \theta, \varphi$ and these variable parameters are related by the two equations

$$
\left\{\begin{array}{l}
e=2 a \cos \theta \\
e=2 h \cos \varphi
\end{array}\right.
$$

Considering that the faces have no thickness, the polyhedron deployment is limited by the following conditions:

- flat folded: when $e=0$ (or $\theta=\varphi= \pm \frac{\pi}{2}$ ).

- fully unfolded: either when $h>a$, the point $O_{2}$ is on $\left[\mathrm{O}_{1} \mathrm{O}_{3}\right]$ leading to $e=2 a$ and $\theta=0$ (fully unfolded prismatic shape condition), or when $h<a$, the point $S_{1}$ is on $\left[H_{1} H_{2}\right]$ leading to $e=2 h$ and $\varphi=0$ (fully unfolded caps condition). When $h=a$, both conditions are met simultaneously.
Self collisions may only occur for concave configurations of the prismatic shape or with re-entrant caps, namely when $b<2 a$ or when $w<2 h$. In this case, self collisions happen when $\theta=-\arcsin \frac{b}{a}$ or $\varphi=\arcsin \frac{w}{h}$. To summarize, the output limits $\left[e_{\min }, e_{\max }\right]$ of the deployable polyhedron can be calculated as:

$$
\begin{aligned}
& e_{\min }=\max \left\{\begin{array}{c}
\sqrt{4 a^{2}-b^{2}}(\text { if } b<2 a) \\
\sqrt{4 h^{2}-w^{2}}(\text { if } w<2 h) \\
0
\end{array}\right. \\
& e_{\max }=\min (2 a, 2 h)
\end{aligned}
$$

\section{PARAMETERS SELECTION}

In order to study and improve the performances of the actuator, the selection of the dimensional parameters is not limited to its mechanical limits. Indeed, several factor have to be accounted for, ranging from the base geometry to considerations related to the implementation of the system.

\section{A. Internal volume}

The analysis of the chamber internal volume gives two characteristics of particular interest in order to predict the behavior of the actuator: i) the fluid flow required in order to actuate the structure, and ii) the inflation (resp. deflation) of the structure reaches a stable configuration when the internal volume reaches a local maximum (resp. minimum). Using both polyhedron parametrization and equations (1), the internal volume can be computed as

$$
\begin{aligned}
V_{\text {act }}= & \overbrace{e w\left(b+\operatorname{sign}(\theta) \sqrt{4 a^{2}-e^{2}}\right)}^{V_{\text {prism }}} \\
& -\operatorname{sign}(\varphi) \underbrace{\frac{e}{6} \sqrt{4 h^{2}-e^{2}}\left(2 b+g+\operatorname{sign}(\theta) \sqrt{4 a^{2}-e^{2}}\right)}_{V_{\text {caps }}}
\end{aligned}
$$

where $V_{\text {prism }}$ and $V_{\text {caps }}$ represent the volume of the prismatic central part of the actuator and the combined volume of both end caps (counted positively). Figure 3 plots the evolution of these volumes in function of the extension $e$, for the selected set of dimensions given in Table I. As expected, $V_{\text {prism }}$ has the most significant influence on the overall volume of the polyhedron. The figure also shows that in the case of a concave prismatic section $(\theta<0$, dashed lines), the volume increases monotonously with respect to the deployment whereas in the case of the convex section $(\theta>0$, solid lines) it reaches a maximum before the full extension $(e=2 a)$, represented by the vertical dashed black line. One should note however that if $\theta$ is allowed to change sign, the actuator should naturally switch configuration from $\theta<0$ and reach the stable state corresponding to the maximum volume when $\theta>0$. Finally, concave configurations for either the prismatic section or the caps also reduce the footprint of the actuator, all other dimensions being equal.

\section{B. Study of the edge of variable length $f$}

As presented in section II-A, in order to allow the actuator's extension, the length $f$ of the four edges $O_{2} S_{1}$ is 


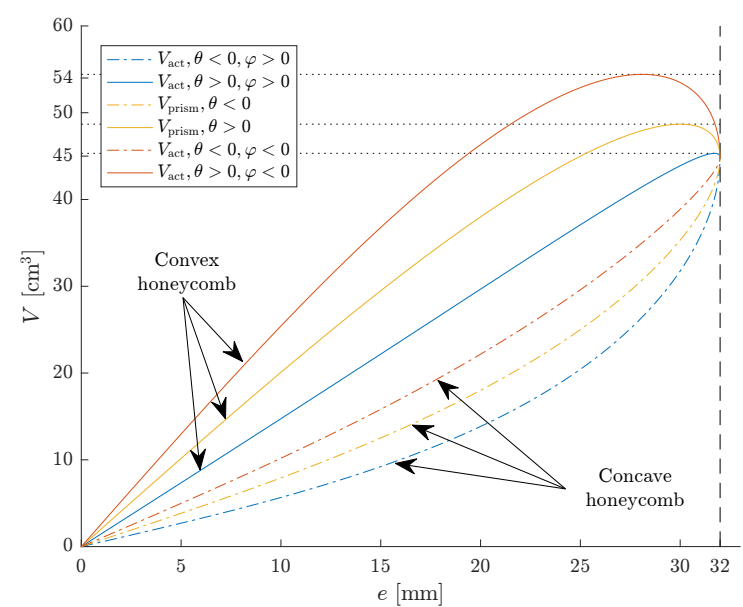

Fig. 3. Evolution of $V_{\text {caps }}$ and $V_{\text {act }}$ with respect to $e$. The evaluation parameters chosen are those of Table I, with negative and positive values for $\theta$ and $\varphi$.
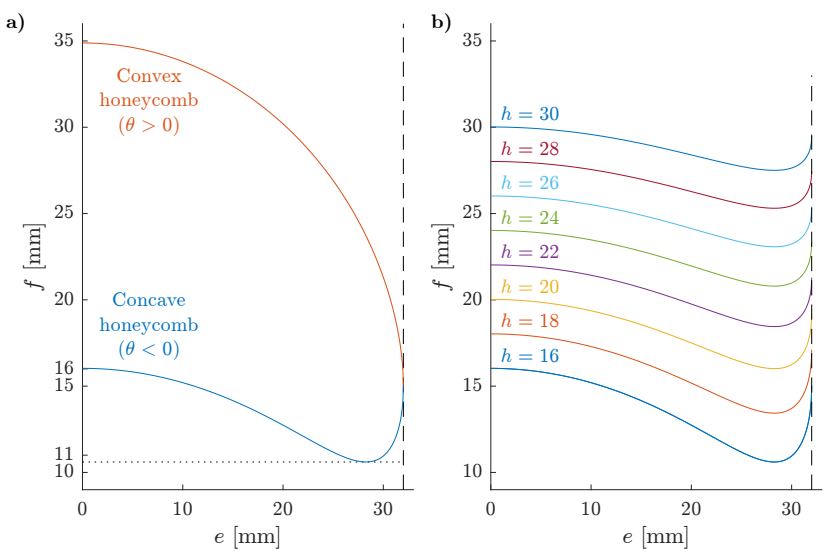

Fig. 4. Evolution of $f$ with respect to $e$ with a) different convexity for the prismatic part, and b) various values for the $h$ parameter considering $\theta<0$. The other evaluation parameters chosen are those of Table I.

assumed to be variable during operation and its expression can be written as

$$
f=\frac{1}{2} \sqrt{4 h^{2}-e^{2}+\left(b-g+\operatorname{sign}(\theta) \sqrt{4 a^{2}-e^{2}}\right)^{2}} .
$$

The figure 4a) shows the evolution of $f$ in function of $e$ for a convex (orange color) and a concave (blue color) shape of the actuator with the parameters of table I. One can see that the variation of $f$ over the range of $e$ is smaller for the convex $(\theta<0)$ configuration. Furthermore, in the practical implementation of these extensible edges, this variation in length will generate traction/compression stress on the material, and combined with a flexion due to the relative rotation of the adjacent faces can lead to the rupture of the internal pocket. Limiting this variation could improve the robustness of the design and therefore the concave $(\theta<$ 0 ) configuration is more desirable. The figure $4 \mathrm{~b}$ ) shows the evolution of $f$ in the concave configuration for different values of $h$ which drive the size of the caps.
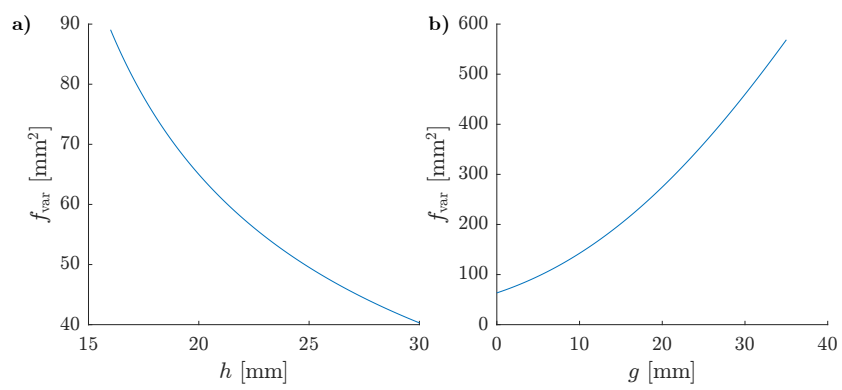

Fig. 5. Evolution of $f_{\text {var }}$ with respect to $e$ and with various values of a) $h$, and b) $g$. The other evaluation parameters used for each plot are those of Table I and $\theta<0$.

In order to define a design criterion, we form the quantity

$$
f_{\mathrm{var}}=\int_{0}^{e_{\max }}\left(f-f_{\min }\right) \mathrm{d} e
$$

where $f_{\min }$ is the minimum of $f$ in the $\left[0 ; e_{\max }\right]$ range. Minimizing $f_{\text {var }}$ leads to a smaller amplitude of variation for $f$ when $e$ varies. The criterion $f_{\mathrm{var}}$ depends on the construction parameters $a, b, g$ and $h$. However, for a given bulkiness of the actuator which is mostly governed by the values of $a$ and $b$, the remaining parameters are $g$ and $h$ and their influence can be explored to minimize $f_{\text {var }}$.

Figures 5a) and 5b) give the plots of $f_{\text {var }}$ as a function of $h$ and $g$ and provide the following design insights:

- higher values for $h$ should be preferred as it also leads to smaller variations of $f$ but this comes at the cost of increasing the footprint of the actuator as it increases the size of the end caps.

- lower values of $g$ leads also to smaller variations of $f$. However, when $g=0, S_{1}$ and $S_{2}$ are coincident, this leads to the usual waterbomb pattern: a 6-th order vertex. Higher order vertices may have a detrimental effect on the resistance of the actuator as they will combine flexions coming from a higher number of edges and thus become a critical point in the structure. This problem is generally tackled by removing the vertex, but as the closure of the volume is mandatory for our application, we opted for low positive values of $g$, creating two 4-th order vertices.

\section{Chosen parameters}

Considering the elements presented in the previous sections, but also other practical limitations imposed by the manufacturing process, including but not limited to precision and material resistance, we have chosen to build the proposed prototype using the parameters indicated in Table. I.

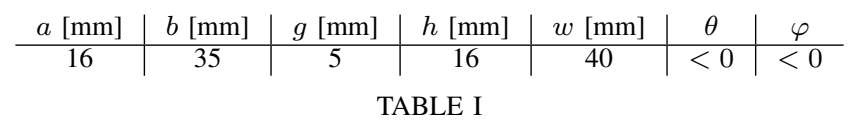

SELECTED PROTOTYPE PARAMETERS.

Although not optimal for limiting the variations of $f, h$ has been chosen small in order to favor compactness. In addition, a parameter named $e_{\text {con }}$ has been added, defining 
the value of $e$ at construction time. This parameter influences the displacement range and also the strain on the hinges of the actuators. Two version are proposed with two different values for $e_{\text {con }}$ : i) Version A built with $e_{\text {con }}=22 \mathrm{~mm}$, and ii) Version B built with $e_{\text {con }}=25 \mathrm{~mm}$. Both versions have been prototyped and evaluated.

\section{PROTOTYPE IMPLEMENTATION}

\section{A. Manufacturing method}

In the last section, the proposed actuator was presented as an ideal model in which most motions are rigid with the only exception of the extensible edges. In this model, the faces were also supposed infinitely stiff and thin. Obviously all of these assumptions cannot be reproduced on an actual prototype where material and manufacturing constraints have to be taken in account. Although several manufacturing methods exist to produce origami-like geometries [24], [25], we chose both for convenience and availability to use a multimaterial additive manufacturing machine in order to produce our prototype.

The machine used, a Connex 350 (Stratasys Ltd. [26]), allows to use a rigid polymer (VeroWhiteplus RGD 835 (VW)) and a "rubber-like" elastomer (TangoBlackplus FLX 980 (TB)), or combinations of both in order to build geometries with varying mechanical properties. This allows the design of prototypes with complex geometries that can be produced with a minimum amount of machining and assembly operations, but at the cost of material performances that are generally inferior to material used in typical molding processes.

\section{B. Flexible origami-like compliant mechanism}

Although the proposed actuator design may work with any kind of fluid, we chose to use compressed air as actuation fluid. Thus, when considering the system, several functions have to be included on the prototype in order to work as initially intended: i) "undeformable" faces should be as rigid as possible, ii) hinges located at the edges and deformable faces have to be as compliant as possible, iii) the internal pocket needs to be airtight and iv) the overall structure should be able to withstand both internal pressure and material deformation. To this end, the CAD model has been created from the geometrical concept by building first a rigid skeleton using $4 \mathrm{~mm}$-thick VW plates and then adding flexures between the faces using $1.5 \mathrm{~mm}$ thick living hinges in $\mathrm{TB}$ to link all rigid faces with their relative neighbors. Deformable faces have also been built in TB, with a thickness of $2 \mathrm{~mm}$ as a trade off between compliance and resistance. The obtained CAD model is presented in Fig. 6.

From this base concept, both the base and end plates have then been cut open to provide an access to the internal room for manufacturing purpose. During assembly phase, two rigid closure caps and a polyurethane inlet tube are glued on the semi-flexible structure in order ensure the internal room tightness and allow exchanges of pressurized air with an external control system.

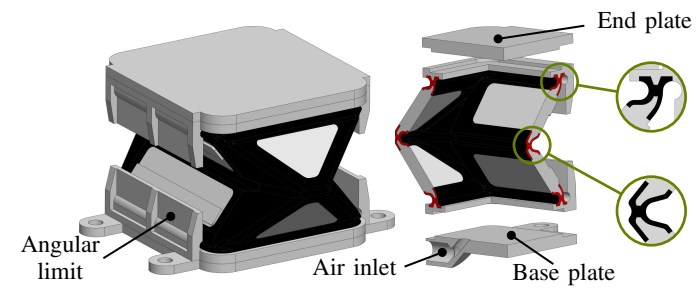

Fig. 6. CAD model of the proposed design (Version B) in full view and quarter section view with detailed views of the hinges implementation. Flexible regions (TB) are represented in black and rigid ones (WV) in white. In red are indicated the flexible regions in the section planes.

\section{Deployment limit}

The inflation of pressurized air in the internal room tends to push the faces of the prismatic section toward the exterior of the actuator, allowing them to fold from a concave to a convex configuration, as it would allow the structure to reach its maximum volume (as discussed in Section III-A). However, this behavior is not desired as: i) it reduces the extension of the actuator and ii) it may increase the stress on the living hinges of the mechanism. Therefore we added on the design stopping geometries (see Fig. 6) in order to limit the rotation of the legs without affecting the deployment of the structure along the $\left(O_{0}, \mathbf{x}\right)$ axis.

\section{Experimental testbed}

During characterization experiments, the prototypes have been controlled using a computer controlled pneumatic testbed as shown in Fig. 7. The input pressure is regulated by a proportional valve (VPPM series, Festo) and a fast 3/2-way solenoid valve (MHE2 series, Festo) triggers the inflation or deflation of the actuator. The pressure of each line is also measured with a pressure sensor (SPTW series, Festo). Displacements of the actuator are then measured by a laser telemeter (optoNCDT 2300 series, Micro-Epsilon) and interaction forces are measured using a $100 \mathrm{~N}$ force sensor (K1563, Scaime) as shown in Fig. 7. Custom software running under Linux Xenomai real-time operating system is used to synchronize all sensor acquisitions with the control of servo-valve and solenoid valves.

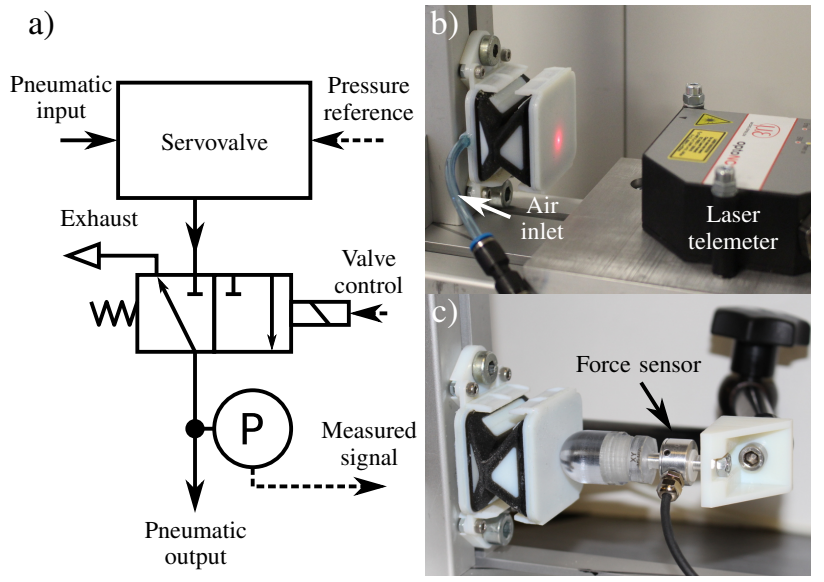

Fig. 7. a) Schematic layout of the pneumatic control testbed. Experimental setup for: b) Displacements measurements, and c) Forces measurements. 


\section{EXPERIMENTAL CHARACTERIZATION}

Before starting the experimental characterization, a preliminary study not presented here has been carried out to assess the maximum pressure before failure of both versions of the proposed actuator. Both versions broke at similar pressure levels, and as such this gave us an estimated safe working pressure of $24.9 \mathrm{kPa}$ that we used as the maximum pressure for all following tests. The minimum pressure level used was determined by the hardware as we could not generate pressure lower than $7.44 \mathrm{kPa}$.

\section{A. Displacement characterization}
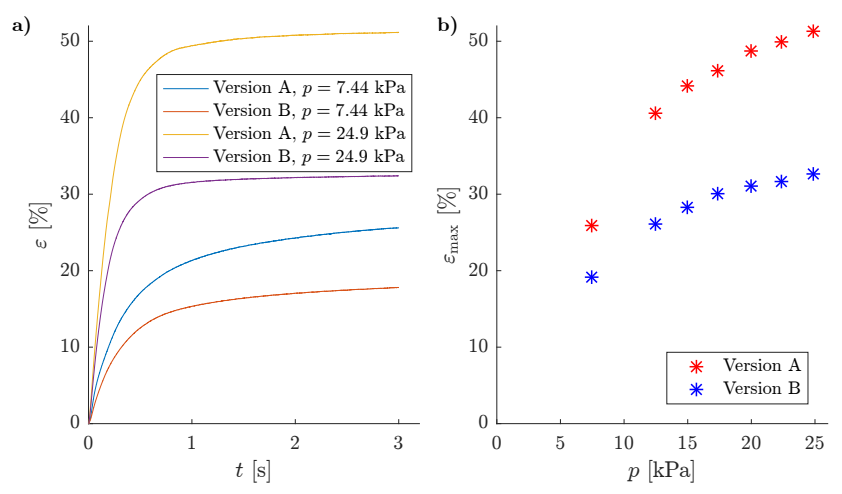

Fig. 8. a) Displacement of both versions of the unloaded actuator at minimum and maximum pressure with respect to time. b) Maximum displacement of the unloaded actuator with respect to different pressures.

The first experiment carried out on the actuator was a displacement characterization. In this test, the actuator was allowed to move freely without load, and several steps of increasing pressures were applied. The results displayed in Fig. 8 show on the left the system transient response for the minimum and maximum pressures, where $\varepsilon=\frac{e-e_{\mathrm{con}}}{e_{\mathrm{con}}}$ represents the deformation relative to the initial size of the actuator (expressed in percentage). On the right is plotted the maximum relative deformation of the actuator at several pressure levels.

The observed deformation show a maximum of $51.3 \%$ (resp. 32.7\%) for the version A (resp. B) at a pressure of $24.9 \mathrm{kPa}$, corresponding to a maximum displacement of about $11.3 \mathrm{~mm}$ (resp. $8.2 \mathrm{~mm}$ ). In both cases, the maximum value for $e$ is superior to its theoretical maximum of $32 \mathrm{~mm}$ (see equation (2)). This phenomenon is due to a deformation along the $\mathbf{x}$ axis of the living hinges that had not be taken in account in the geometric model.

\section{B. Force characterization}

The second experiment carried out on the actuator was a static force characterization. In this test, the extension of the actuator was blocked by a force sensor in order to measure the force generated by the system in its constructed position under several steps of pressure. Indeed as only a limited amount of energy is stored in material elasticity, the force generated at the constructed configuration should be maximal. The results displayed in Fig. 9 show on the left the transient response of the system for the minimum and
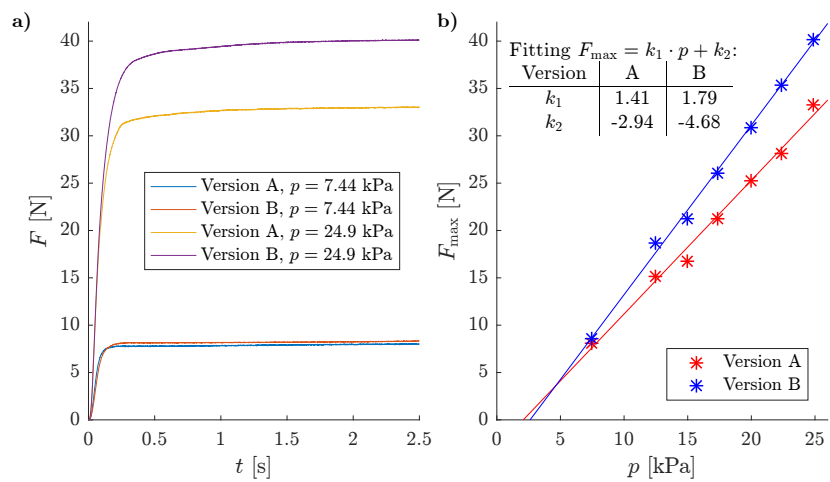

Fig. 9. a) Static force response generated by both versions of the actuator at minimum and maximum pressure with respect to time. b) Maximum force generated by the actuator the actuator with respect to different pressures.

maximum pressure. On the right is plotted the maximum force observed at each pressure levels.

At low pressure, both systems generate similar levels of force, but as the pressure raises, the difference between both versions of the system increases. At the maximum pressure, version $\mathrm{A}$ is able to generate $33.2 \mathrm{~N}$ (resp. $40.0 \mathrm{~N}$ for version B). By comparison, a frictionless cylinder with a similar footprint $\left(14 \mathrm{~cm}^{2}\right.$ for the base of the actuator) would generate a force of $34.9 \mathrm{~N}$ at $24.9 \mathrm{kPa}$.

\section{Cyclic loading}

Both of the previous experiments have been carried out by using only one pressure step for each pressure level. In order to characterize the repeatability and the resistance of the system, a cyclic test has been performed. Both displacement and force generation have been tested using a periodic step pressure load with a period of approximately 8s (due to software limitation, the triggering was performed manually). The selected pressure for this test was $17.4 \mathrm{kPa}$ in order to limit the stress on the structure while generating significant displacements and forces.

\begin{tabular}{c||c|c||c|c}
\multicolumn{1}{c||}{} & \multicolumn{2}{c||}{ Max. deformation [\%] } & \multicolumn{2}{c}{ Max. force [N] } \\
\hline Version & Mean & Std. & Mean & Std. \\
\hline A & 47.2 & 0.48 & 24.4 & 0.44 \\
B & 29.7 & 0.18 & 27.1 & 0.34 \\
\multicolumn{1}{c|}{ TABLE II }
\end{tabular}

CYCLIC LOADING PERFORMANCES AT 17.4 KPA.

Both versions of the prototype have been tested and the displacement (or forces) were measured at each cycle. Table II shows the mean and standard deviation of the maximum forces and displacements measured during the tests. Although version A (resp. B) broke after 26 (resp. 30) cycles during the cyclic displacement test, both version were still in working condition after more than 60 cycles in the force tests. This difference is probably due to the lower level of material deformation during force tests with respect to displacements tests. 


\section{Experimental results discussion}

In parallel to the quantitative results shown in this section, some observations can be made on the overall experimental assessment. No dynamic experiment have been carried out as they are influenced by both the actuator and the air supply performance. It has been observed however that at lower working pressures, the air pressure supplied by the testbed rises with an unrepeatable profile, leading some experimental results that were discarded. Changing the servo-valve for a better suited model should improve further experiments. Testing the off-axis stiffness and on-axis force displacement relationship would also be of interest. It would however require a more complex setup than currently available as forces need to be measured while the displacement are constrained (or reciprocally) along several directions. Finally, a limitation of the manufacturing process has also been observed during the tests. The MMAM process used creates ridges aligned with the horizontal plane of printing. These fracture initiations, combined with mixed tensile and flexural deformations along the living hinges, drastically limit the lifetime of the actuator.

\section{Conclusions}

In this paper, a new robotic actuator was proposed, based on a monolithic design combining both stiff and soft elements. Two variants have been built and their performances have been assessed for both displacements and force generation. Forces in the ranges of $40 \mathrm{~N}$ at a pressure of $24.9 \mathrm{kPa}$ were measured as well as relative deformations without load in the order of $51.3 \%$ starting from the initial actuator size. Although both versions of the prototype exhibit a limited number of work cycles, especially at higher deformation ranges, they perform with a decent robustness and repeatability considering the limitations of the manufacturing process.

Further work will focus on improving the current design by trying to reduce extension at the living hinges in order 1) to increase their resistance; 2) to be closer to the theoretical model of the system, improving the system mechanical accuracy. In order to solve this issue we will adapt the present concept to other manufacturing methods such as overmolding on composite structure, in order to combine fully rigid parts, flexible fibrous materials and elastomers. Improvements of the testbed are also required in order to carry out more indepth assessments of the system.

The proposed prototype is also only capable of producing forces and displacements in one direction. We will work on implementing a bidirectional system by either using two actuators in antagonistic configurations, or by implementing a positive/negative pressure control with a single actuator in order to favor a more compact actuation solution.

\section{REFERENCES}

[1] A. A. Calderón, J. C. Ugalde, J. C. Zagal, and N. O. Pérez-Arancibia, "Design, fabrication and control of a multi-material-multi-actuator soft robot inspired by burrowing worms," in 2016 IEEE International Conference on Robotics and Biomimetics (ROBIO), Dec. 2016, pp. 31-38.

[2] N. Correll, Ç. D. Önal, H. Liang, E. Schoenfeld, and D. Rus, "Soft Autonomous Materials-Using Active Elasticity and Embedded Distributed Computation," SpringerLink, pp. 227-240, 2014.
[3] J. M. Florez, B. Shih, Y. Bai, and J. K. Paik, "Soft pneumatic actuators for legged locomotion," in 2014 IEEE International Conference on Robotics and Biomimetics (ROBIO 2014), Dec. 2014, pp. 27-34.

[4] M. T. Tolley, R. F. Shepherd, M. Karpelson, N. W. Bartlett, K. C. Galloway, M. Wehner, R. Nunes, G. M. Whitesides, and R. J. Wood, "An untethered jumping soft robot," in 2014 IEEE/RSJ International Conference on Intelligent Robots and Systems, Sep. 2014, pp. 561566.

[5] S. Terryn, G. Mathijssen, J. Brancart, D. Lefeber, G. V. Assche, and B. Vanderborght, "Development of a self-healing soft pneumatic actuator: A first concept," Bioinspiration \& Biomimetics, vol. 10, no. 4, p. $046007,2015$.

[6] M. A. Robertson, H. Sadeghi, J. M. Florez, and J. Paik, "Soft Pneumatic Actuator Fascicles for High Force and Reliability," Soft Robotics, vol. 4, no. 1, pp. 23-32, Oct. 2016.

[7] A. Stilli, H. A. Wurdemann, and K. Althoefer, "A Novel Concept for Safe, Stiffness-Controllable Robot Links," Soft Robotics, vol. 4, no. 1, pp. 16-22, Mar. 2017.

[8] J. Hiller and H. Lipson, "Automatic Design and Manufacture of Soft Robots," IEEE Transactions on Robotics, vol. 28, no. 2, pp. 457-466, Apr. 2012.

[9] A. Bruyas, F. Geiskopf, and P. Renaud, "Toward unibody robotic structures with integrated functions using multimaterial additive manufacturing: Case study of an MRI-compatible interventional device," in 2015 IEEE/RSJ International Conference on Intelligent Robots and Systems (IROS), Sep. 2015, pp. 1744-1750.

[10] Y. Sun, Y. S. Song, and J. Paik, "Characterization of silicone rubber based soft pneumatic actuators," in 2013 IEEE/RSJ International Conference on Intelligent Robots and Systems, Nov. 2013, pp. 44464453.

[11] B. Mosadegh, P. Polygerinos, C. Keplinger, S. Wennstedt, R. F. Shepherd, U. Gupta, J. Shim, K. Bertoldi, C. J. Walsh, and G. M. Whitesides, "Pneumatic Networks for Soft Robotics that Actuate Rapidly," Advanced Functional Materials, vol. 24, no. 15, pp. $2163-$ 2170, Apr. 2014.

[12] F. Ilievski, A. D. Mazzeo, R. F. Shepherd, X. Chen, and G. M. Whitesides, "Soft Robotics for Chemists," Angewandte Chemie International Edition, vol. 50, no. 8, pp. 1890-1895, Feb. 2011.

[13] K. Miura and T. Tachi, "Synthesis of rigid-foldable cylindrical polyhedral," Symmetry: Art and Science, International Society for the Interdisciplinary Study of Symmetry, Gтиеnd, 2010.

[14] T. Tachi and K. Miura, "Rigid-foldable cylinders and cells," J. Int. Assoc. Shell Spat. Struct, vol. 53, no. 4, pp. 217-226, 2012.

[15] E. T. Filipov, T. Tachi, and G. H. Paulino, "Origami tubes assembled into stiff, yet reconfigurable structures and metamaterials," Proceedings of the National Academy of Sciences, vol. 112, no. 40, pp. 12 321-12 326, Jun. 2015.

[16] T. Liu, Y. Wang, and K. Lee, "Three-Dimensional Printable Origami Twisted Tower: Design, Fabrication, and Robot Embodiment," IEEE Robotics and Automation Letters, vol. 3, no. 1, pp. 116-123, Jan. 2018.

[17] S. Li and K. W. Wang, "Fluidic origami: A plant-inspired adaptive structure with shape morphing and stiffness tuning," Smart Materials and Structures, vol. 24, no. 10, p. 105031, Oct. 2015.

[18] D. Kim and R. B. Gillespie, "Origami Structured Compliant Actuator (OSCA)," in 2015 IEEE International Conference on Rehabilitation Robotics (ICORR), Aug. 2015, pp. 259-264.

[19] S. Li, D. M. Vogt, D. Rus, and R. J. Wood, "Fluid-driven origamiinspired artificial muscles," Proceedings of the National Academy of Sciences, vol. 114, no. 50, pp. 13 132-13 137, Nov. 2017.

[20] L. Paez, G. Agarwal, and J. Paik, "Design and Analysis of a Soft Pneumatic Actuator with Origami Shell Reinforcement," Soft Robotics, vol. 3, no. 3, pp. 109-119, Sep. 2016.

[21] C. D. Onal, R. J. Wood, and D. Rus, "An Origami-Inspired Approach to Worm Robots," IEEE/ASME Transactions on Mechatronics, vol. 18, no. 2, pp. 430-438, Apr. 2013.

[22] C. Qiu, K. Zhang, and J. S. Dai, "Repelling-Screw Based Force Analysis of Origami Mechanisms," Journal of Mechanisms and Robotics, vol. 8, no. 3, p. 031001, Mar. 2016.

[23] A. Connelly, R., Sabitov, and I., Walz, "The Bellows conjecture." Beiträge zur Algebra und Geometrie, vol. 38, no. 1, pp. 1-10, 1997.

[24] R. V. Martinez, C. R. Fish, X. Chen, and G. M. Whitesides, "Elastomeric Origami: Programmable Paper-Elastomer Composites as Pneumatic Actuators," Advanced Functional Materials, vol. 22, no. 7, pp. 13761384, Apr. 2012.

[25] Z. Zhakypov and J. Paik, "Design Methodology for Constructing Multimaterial Origami Robots and Machines," IEEE Transactions on Robotics, vol. 34, no. 1, pp. 151-165, Feb. 2018.

[26] Stratasys, Polyjet Process. 